\title{
Social Media - History and Components
}

\author{
${ }^{1}$ Sajithra K, ${ }^{2}$ Dr. Rajindra Patil
}

\section{Social Media Overview}

\section{Introduction}

Social Media is an extension and explosion of traditional word of mouth networks. Word of mouth has always been the most effective and trust worthy means of disseminating information. With the enablement of technology, anybody with an internet access and has an opinion can be part of social media. This cultural shift is a force to reckon with for companies.

There were more than 500 million active users on Facebook, 70 percent outside the United States in 2010. ${ }^{1}$ By March 2010, more than 10 billion messages, or Tweets, had been sent through Twitter since its launch in 2006. By July, that number had doubled to 20 billion. ${ }^{2} 50$ percent of the total online population visited a social networking site in February 2010 in the Asia-Pacific region,, reaching a total of 240.3 million visitors. ${ }^{3}$ Social Media is conversation online and cannot be ignored since the customers, investors, critics, fans and competition are conversing in a medium that can be easily manipulated!

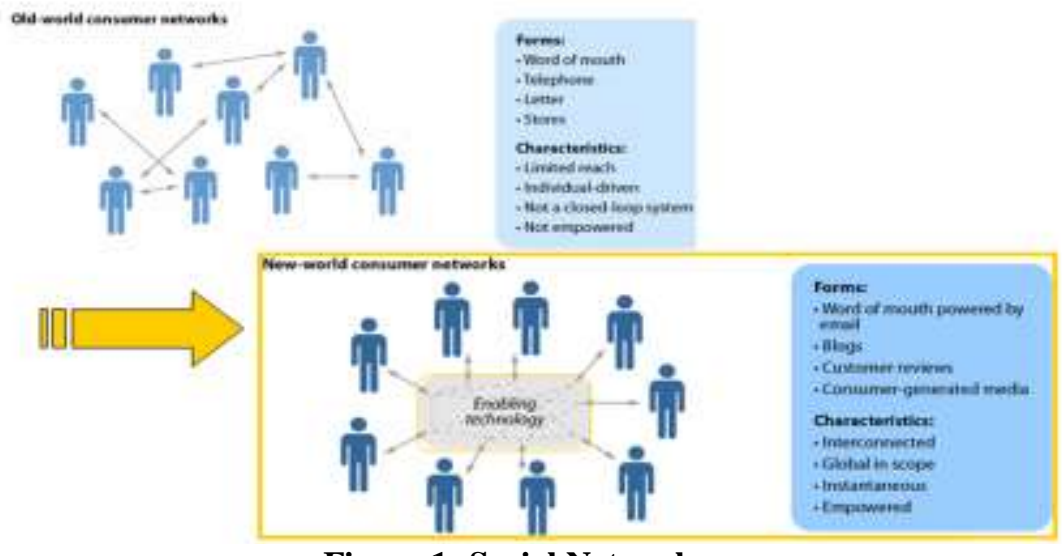

Figure 1: Social Networks

\section{History of Social Media}

It is important to understand the history of a phenomenon in order to manipulate it. At first glance, Social Media comes across as novelty. But a careful evaluation helps the user to trace back the origins of Social Media.

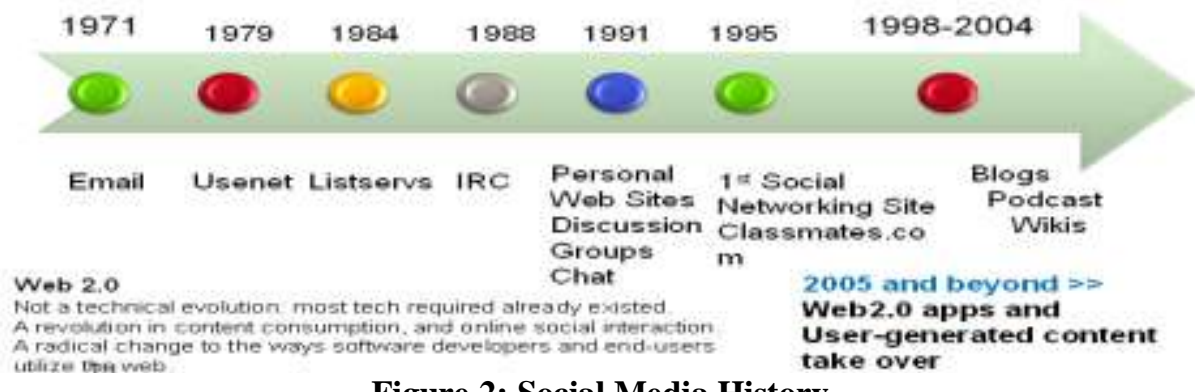

Figure 2: Social Media History

\footnotetext{
1 “Statistics." Press room. Facebook Web site, accessed January 10, 2011. http://www.facebook.com/press/info.php?statistics

${ }^{2}$ Beaumont, Claudine. "Twitter hits 10 billionth tweet." The Telegraph. March 5, 2010.

3 "Social Networking Habits Vary Considerably Across Asia-Pacific Markets." comScore press release. April 7, 2010.
} 


\section{1: Email}

There are ongoing debates on whether Email could be considered a part of social media. The predominant reasons why Email is not considered a social media are because (i) Email is a distribution mechanism whereas Social Media is a collective mechanism and (ii) Mass communication is different from Mass collaboration. ${ }^{4}$ But Email certainly qualifies if we go by the simple definition that 'social media is conversations that happen online'.

Though the debate goes on, we cannot ignore the fact that the introduction of Email marked the beginning to the much more collaborative social media years later.

Computer engineer, Ray Tomlinson invented internet based email in late 1971. Ray Tomlinson worked as a computer engineer for Bolt Beranek and Newman (BBN), the company hired by the United States Defense Department to build the first Internet in 1968.

Ray Tomlinson along with a team was developing a time-sharing system called TENEX that ran on Digital PDP-10 computers. They were supporting a larger group working on natural language. Earlier, Ray Tomlinson had worked on the Network Control Protocol (NCP) for TENEX and network programs such as an experimental file transfer program called CPYNET.

Ray Tomlinson was making improvements to the local inter-user mail program called SNDMSG. Single-computer electronic mail had existed since at least the early 1960's and SNDMSG was an example of that. SNDMSG allowed a user to compose, address, and send a message to other users' mailboxes. The mail box was a file that could be appended but not overwritten. Ray Tomlinson tied his previous experience with CPYNET, a file transfer program with SNDMSG, a file that could be appended and came up with the idea of email, sending direct messages to remote mail boxes in addition to appending messages to local mail boxes.

SNDMSG that the ARPANET programmers and researchers were using on the network computers (Digital PDP-10s) allowed them to leave messages for each other. SNDMSG was a "local" electronic message program. A person could only leave messages on the computer for other persons using that same computer. Tomlinson used the file transfer protocol from CYPNET to adapt the SNDMSG program so it could send electronic messages to any other computer on the ARPANET. The first message was sent between two machines that were literally side by side. The only physical connection they had (aside from the floor they sat on) was through the ARPANET. The first use of network email announced its own existence.

These first messages were sent in late 1971. The next release of TENEX went out in early 1972 and included the version of SNDMSG with network mail capabilities. The CPYNET protocol was soon replaced with a real file transfer protocol having specific mail handling features. Later, a number of more general mail protocols were developed. ${ }^{5}$

\section{9: Usenet}

Usenet is a worldwide distributed Internet discussion system. It developed from the general purpose UUCP architecture of the same name.

Duke University graduate students Tom Truscott and Jim Ellis initiated this in 1980. They improvised the Email concept to share categorized messages. Users could read and post messages to one or more categories. These groups were known as newsgroups.

Usenet was founded based on a necessity. Tom Truscott and Jim Ellis tried to replace the existing BBB style announcement system which became obsolete with a recent software upgrade in their university.

Usenet worked on constantly changing collection of servers that store and forward messages to one another in so-called news feeds. This is different from a BBS or web forum hosted from a central server and dedicated administrator. Individual users may read messages from and post messages to a local server operated by their own Internet service provider, university, or employer. Steve Bellowin assisted in writing the scripts and their 'net news program linked Duke and University of North Carolina. This software was then made available to the general public as 'A News'.

\footnotetext{
${ }_{5}^{4}$ Source: Anthony Bradley, Gartner

${ }^{5}$ Source: http://openmap.bbn.com

${ }^{6}$ Source: http://www.giganews.com/usenet-history/index.html
} 


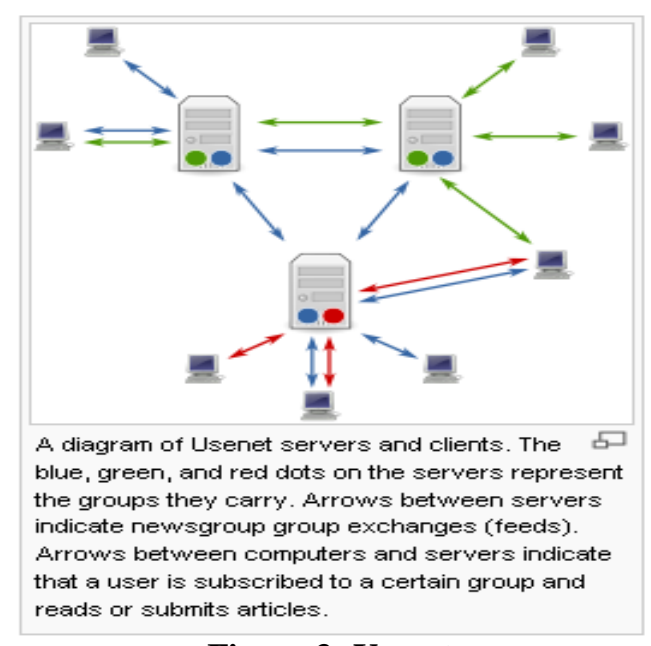

Figure 3: Usenet

\section{4: LISTSERV}

LISTSERV scaled up the usage of email communication and proved as an effective way to reach out to a large number of people in an instant.

Prior to LISTSERV, email lists were managed manually. People would have to write to the administrator who manages the list and ask to be added or removed. This process only got more time-consuming as discussion lists grew in popularity.

Eric Thomson, an engineering student tried to automate the process of managing email lists. Eric Thomson's email list management program, known as LISTSERV became a huge success.

The emailing mechanism was expanded with the introduction of LISTSERV. LISTSERV was the first electronic mailing list software application, in which the sender can send one email and it will reach a group of people. After the launch of LISTSERV in 1986, several other list management tools have been developed, such as Lyris ListManager in 1997, Sympa in 1997, GNU Mailman in 1998.

LISTSERV was freeware from 1986 through 1993. It is now a commercial product developed by LSoft, a company founded by LISTSERV author Eric Thomas in 1994.

\section{8: IRC}

Email, Usenet and Listserv helped in sharing messages, individually, categorized and also to a group through a list. This still could not replicate the ease of meeting a person face to face. Internet Relay Chat (IRC) introduced in 1988 improved the experience. IRC is a form of chatting. Originally designed for group chatting in discussion forums, this also allowed one-to-one communication via private message as well as chat and data transfers.

As of May 2009, the top 100 IRC networks served more than half a million users at a time, with hundreds of thousands of channels (the vast majority of which stand mostly vacant), operating on roughly 1,500 servers worldwide. ${ }^{8}$ (Jones, 2002-12-10)

IRC was created by Jarkko Oikarinen in August 1988 to replace a program called MUT (MultiUser Talk) on a BBS called OuluBox in Finland. Oikarinen was inspired by chat system known as Bitnet Relay. Jarkko Oikarinen was working in the Department of Information Processing Science in University of Oulu as an administrator for the Sun Server and started working on a communications program during his free time. He wanted to include USENET News kind of systems to the regular real time discussions in their BBS system OuluBox and succeeded. ${ }^{9}$

IRC was used to report on the 1991 Soviet coup d'état attempt throughout a media blackout. It was previously used in a similar fashion during the Gulf War. Logs of these and other events are kept in the ibiblio archive. $^{10}$

IRC client software is available for nearly every computer operating system that supports TCP/IP networking.

\footnotetext{
${ }^{7}$ Source: http://www.lsoft.com/corporate/history-listserv.asp

${ }^{8}$ Jones, Steve, ed. (2002-12-10). "Internet Relay Chat". Encyclopedia of New Media: An Essential Reference to Communication and Technology (1st ed.)

${ }^{9}$ Source: www.irc.org

${ }^{10}$ IRC logs of events of the Gulf War. Chapel Hill, North Carolina: ibiblio. Retrieved 2011-04-08
} 


\section{1: Personal websites, Discussion groups, chat}

After the success of IRC, many personal websites, discussion groups and chat groups also became popular. At the beginning of the 90s, internet access was not completely accessible to the public. This situation changed when Private internet service providers (ISPs) began to start operations in the United States around 1994 or 1995. This gave millions of home users the chance to experience it. The other reason for the initial euphoria was the fact that the content was absolutely free other than the data usage paid to the internet companies. Early internet users were extremely outspoken and opinionated by today's standards. People were thrilled at the possibility of sharing their opinions and often went overboard in their expressions. The first online social media etiquette standards were proposed, and called netiquette, as a control mechanism. Internet forums grew in popularity by the late 90's and began replacing Usenet and BBSes as the primary platform for topical discussions.

\section{5: Social Networking site classmate.com}

Randy Conrads created Classmates.com in 1995 by. Nielsen Online ranked Classmates as number three in unique monthly visitors (U.S. home, work) among social networking sites in 2008. The objective of this social media website is to assist members in finding friends and acquaintances from throughout their lives, from kindergarten, primary school, high school, college, work and the United States military. Classmates.com has over 50 million members.

\section{8-2004}

Blogs, Podcast, Wikis

The modern blog evolved from the online diary. Justin Hall, who began personal blogging in 1994 is generally recognized as one of the earliest bloggers. ${ }^{11}$ The other popular blogs are Dave Winer's Scripting News and Wearable Wireless Webcam. Wearable Wireless Webcam was unique in terms of how it combined text, video, and pictures transmitted live from a wearable computer and EyeTap device to a web site in 1994. This practice of semi-automated blogging with live video together with text was referred to as sousveillance. Such entries were considered as legal evidence as well.

Early blogs were simply updates in common Web sites. However, the evolution of tools to facilitate the production and maintenance of Web articles posted in reverse chronological order made the publishing process feasible to a much larger, less technical, population. Ultimately, this resulted in the distinct class of online publishing that produces blogs we recognize today. For instance, the use of some sort of browser-based software is now a typical aspect of "blogging". Blogs can be hosted by dedicated blog hosting services, or they can be run using blog software, or on regular web hosting services. Blogs are recognized as a separate medium in itself.

Some early bloggers, such as The Misanthropic Bitch, who began in 1997, actually referred to their online presence as a zine, before the term blog entered common usage.

After a slow start, blogging rapidly gained in popularity. Blog usage spread during 1999 and the years following, being further popularized by the near-simultaneous arrival of the first hosted blog tools:

* Bruce Ableson launched Open Diary in October 1998. Open Diary was the first in allowing the readers to add comments to the blog entries. ${ }^{12}$

* Brad Fitzpatrick started LiveJournal in March 1999.

* Andrew Smales created Pitas.com in July 1999 as an easier alternative to maintaining a "news page" on a Web site, followed by Diaryland in September 1999, focusing more on a personal diary community.

* Evan Williams and Meg Hourihan (Pyra Labs) launched blogger.com in August 1999 (purchased by Google in February 2003)

\section{5 and beyond}

Web2.0 applications and user generated content took over during this time period:-

The term Web 2.0 is commonly associated with web applications that facilitate interactive information sharing, interoperability, user-centered design, and collaboration on the World Wide Web. A Web 2.0 site gives its users the free choice to interact or collaborate with each other in a social media dialogue as creators (prosumer) of user-generated content in a virtual community, in contrast to websites where users (consumer) are limited to the passive viewing of content that was created for them. Examples of Web 2.0 include socialnetworking sites, blogs, wikis, video-sharing sites, hosted services, web applications, mashups and folksonomies.

\footnotetext{
${ }^{11}$ Source: Harmanci, Reyhan (2005-02-20). "Time to get a life — pioneer blogger Justin Hall bows out at 31". San Francisco Chronicle. Retrieved 2008-06-05.

12 Source : Gaudeul, Alexia and Peroni, Chiara (2010). "Reciprocal attention and norm of reciprocity in blogging networks". Economics Bulletin 30 (3): 2230-2248
} 
The term is closely associated with Tim O'Reilly because of the O'Reilly Media Web 2.0 conference in 2004. Although the term suggests a new version of the World Wide Web, it does not refer to an update to any technical specifications, but rather to cumulative changes in the ways software developers and end-users use the Web. Whether Web 2.0 is qualitatively different from prior web technologies has been challenged by World Wide Web inventor Tim Berners-Lee, who called the term a "piece of jargon", precisely because he intended the Web in his vision as "a collaborative medium, a place where we [could] all meet and read and write". He called it the 'Read/Write Web'.

\section{Web 3.0}

Amit Agrawal describes web3.0 as the Semantic Web and personalization. Conrad Wolfram has a computer based approach to web 3.0 that web3.0 is about the computer generating new information instead of humans. Today, the problem is not lack of information but too much information. The challenge is making sense of this information and categorizing it appropriately. Web3.0 is more about the tools and techniques to make this information consumption efficient and effective.

Manoj Sharma, an organization strategist, in the keynote "A Brave New World Of Web 3.0" proposes that Web 3.0 will be a "Totally Integrated World" - cradle-to-grave experience of being always plugged onto the net. Andrew Keen, author of The Cult of the Amateur, considers the Semantic Web an "unrealisable abstraction". He points out Bertelsman's deal with the German Wikipedia to produce an edited print version of that encyclopedia. CNN Money's Jessi Hempel speculates Web 3.0 to emerge from new and innovative Web 2.0 services with a profitable business model.

John Smart, author of the Metaverse Roadmap echoes Sharma's perspective that web3.0 will bring out a completely integrated world, defining Web 3.0 as the first-generation Metaverse (convergence of the virtual and physical world), a web development layer that includes TV-quality open video, 3D simulations, augmented reality, human-constructed semantic standards, and pervasive broadband, wireless, and sensors. Web 3.0's early geosocial (Foursquare, etc.) and augmented reality (Layar, etc.) webs are an extension of Web 2.0's participatory technologies and social networks (Facebook, etc.) into 3D space. Of all its metaverse-like developments, Smart suggests Web 3.0's most defining characteristic will be the mass diffusion of NTSC-or-better quality open video to TVs, laptops, tablets, and mobile devices, a time when "the internet swallows the television."Smart considers Web 4.0 to be the Semantic Web and in particular, the rise of statistical, machine-constructed semantic tags and algorithms, driven by broad collective use of conversational interfaces, perhaps circa 2020.

David Siegel's perspective in Pull: The Power of the Semantic Web, 2009 proposes that the growth of human-constructed semantic standards and data will be a slow, industry-specific incremental process for years to come, perhaps unlikely to tip into broad social utility until after 2020 .

Social Media History points out how the entire phenomenon relied on power of the crowd. The technological Innovation had more to do with how a certain technology was combined with something else to bring in the power of social groups than a break through Innovation. Email was only an incremental Innovation of an existing program that let people leave messages on their personal computer. When that was combined with the file transfer program through ARPANET, the scope of reaching people expanded and ultimately resulted in a disruptive innovation.

True to its history, Social Media impact also relies on how well digital technologies are matched with the goal of the company and the need of the target audience.

\section{Components of Social Media}

Social media thus is an evolution of word of mouth that scaled up by leveraging the pervasiveness of Internet. The major components of social media span are 1) Social Networking 2) Micro blogs 3) Blogs 4) RSS Feeds 5) Widgets 6) Linking and posting 7) Content Rating 8) Bookmarking sites 9) Audio podcasting 10) Video podcasting 


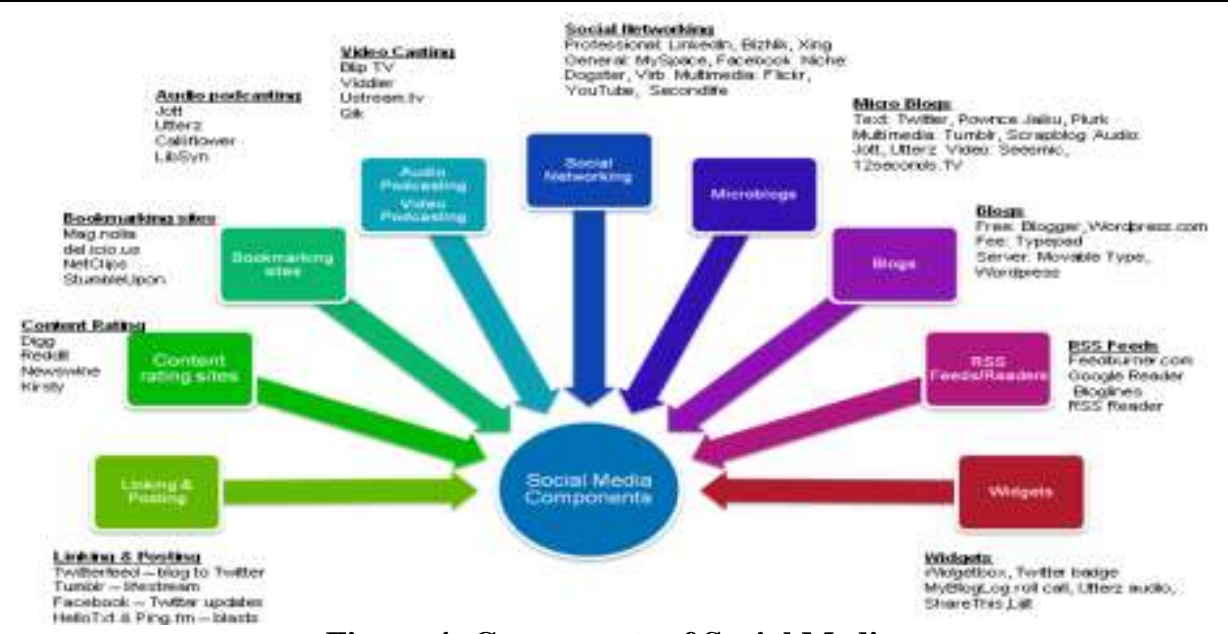

Figure 4: Components of Social Media

This paper tracks the History of Social Media and tabulates the various components of Social Media. The study concludes that Social Media is an explosion of word of mouth networks and is different from Digital Marketing that does not always rely on social interactions. Utilizing Social Media for Marketing is an inorganic means of building Reach, Engagement and Intelligence whereas Digital Marketing involves Social Media and also the usage of tactics like PPM, banner displays, Online Advertising, Mobile Adverts etc. 